\title{
Neuroprotective Effect of Z-Ligustilide against Permanent Focal Ischemic Damage in Rats
}

\author{
Hai-Yan Peng, ${ }^{a}$ Jun-Rong Du, ${ }^{*}, a$ Guang-Yi Zhang, ${ }^{a}$ Xi KuAnG, ${ }^{a}$ Yan-Xin Liu, ${ }^{a}$ \\ Zhong-Ming QIAN, ${ }^{b}$ and Chen-Yuan $\mathrm{WANG}^{b}$ \\ ${ }^{a}$ Department of Pharmacology, West China School of Pharmacy, Sichuan University; Chengdu, 610041 P.R. China: and \\ ${ }^{b}$ Department of Applied Biology \& Chemical Technology, Hong Kong Polytechnic University; Hong Kong. \\ Received September 19, 2006; accepted November 18, 2006
}

\begin{abstract}
The present study investigated the effect of Z-Ligustilide (LIG), a characterized 3-n-alkyphthalide derivative existed in many medical Umbelliferae plants, on permanent focal ischemic brain injury in rats. Focal cerebral ischemia was induced by the occlusion of middle cerebral artery (MCA) for $24 \mathrm{~h}$. LIG (20, or $80 \mathrm{mg} / \mathrm{kg}$ ), orally administered at $2 \mathrm{~h}$ after ischemia, reduced the cerebral infarct volumes by $48.29 \%$ and $84.87 \%$ respectively compared to control group as visualized by $2,3,5$-triphenyltetrazolium chloride (TTC) staining $(p<0.01)$. Treatment with LIG could dose-dependently reduce brain swelling by $68.62 \%$ and $82.08 \%(p<0.01)$, and significantly improve behavioral deficits $(p<0.01)$. In addition, LIG at the above used doses had no significant effect on rat body temperature. These data, along with previous findings in our lab demonstrating the neuroprotective effects of LIG in transient cerebral ischemia, suggest that LIG may be a potential neuroprotective agent for the treatment of ischemic stroke in future.
\end{abstract}

Key words Ligustilide (LIG); 3- $n$-alkyphthalide derivative; Radix Angelica sinensis (Danggui); focal cerebral ischemia; brain edema; neuroprotective agent

Stroke remains one of the major causes of death and disability throughout the world. More than $80 \%$ of all strokes are a result of cerebral ischemia. Approximately $20 \%$ of stroke patients do not survive the first month and less than $30 \%$ of those who survive the first six months would become dependent on others. ${ }^{1)}$ In correlation with the socio-economic importance of ischemic stroke, neuroprotective agents with different modes of action and an extended application time window are therefore urgently required in clinic.

Radix of Angelica sinensis, known as Danggui in Chinese, has been used to treat cardiovascular and cerebrovascular diseases in traditional Chinese medicines (TCM) for a long time. Numerous studies indicate that the effective constituents of Danggui are classified into water soluble part and essential oil. And the former including ferulic acid and polysaccharides is widely used in clinic, while the effective substance basis of the essential oil is not completely clear. ${ }^{2)} \mathrm{Re}-$ cently, Z-Ligustilide (LIG, Fig. 1), the main lipophilic component of Danggui, ${ }^{3)}$ was isolated and purified from the essential oil in our lab. Our previous studies showed that LIG significantly prevented transient ischemic brain damage., ${ }^{4,5)}$ The oral administration of LIG $(20$, or $80 \mathrm{mg} / \mathrm{kg})$, at 0 and $4 \mathrm{~h}$ again after reperfusion following $2 \mathrm{~h}$ ischemia, significantly reduced infarct volumes and improved neurological scores in transient middle cerebral artery (MCA) occlusion rats. ${ }^{5)}$ In addition, LIG $(5$, or $20 \mathrm{mg} / \mathrm{kg}$, i.p.) could also remarkably reduce the infarct volume in the transient forebrain ischemic mice in a dose-dependent manner. $\left.{ }^{4}\right)$ It is reported

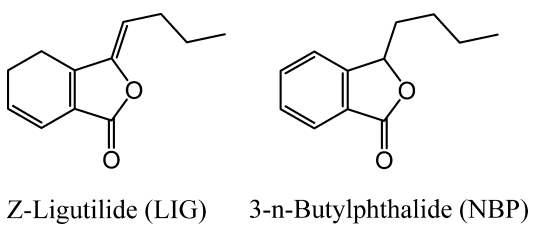

Fig. 1. Chemical Structures of Z-Ligustilide (LIG) and 3- $n$-Butylphthalide (NBP)

* To whom correspondence should be addressed. e-mail: dujr_1@163.com that the cerebral ischemia due to lasting vascular blockage plays a more important role in clinical ischemic stroke. ${ }^{6)}$ In order to further investigate the protection of LIG on focal brain ischemia, the present study therefore evaluated its effect in rat permanent MCA occlusion model. The effect of LIG on infarct volumes, edema, neurological scores and body temperature was assessed in this study.

\section{MATERIALS AND METHODS}

Preparation of Animals All animal experiments were carried out in accordance with the National Institutes of Health guidelines for the care and use of laboratory animals, and provided by the Center of Experimental Animal in Sichuan University.

Male Sprague-Dawley rats weighing 250 to $300 \mathrm{~g}$ were anesthetized with chloral hydrate (Tianjin, P.R. China) intraperitoneally at a dose of $300 \mathrm{mg} / \mathrm{kg}$. Permanent focal brain ischemia model was induced by the occlusion of MCA by the described procedure. ${ }^{7)}$ Briefly, 4-0 monofilament nylon suture with a round tip was advanced from external carotid artery into the lumen of the internal carotid artery to occlude the origin of the MCA. Throughout the surgery, body temperature was monitored by a rectal probe and maintained at $37 \pm 0.5^{\circ} \mathrm{C}$ by using a heating pad. After the surgical procedures, the rats were returned to their cages in a room where the temperature was maintained 24 to $26^{\circ} \mathrm{C}$.

Pharmacological Treatment LIG was isolated from essential oil of Radix Angelica sinensis by silica-gel column chromatography and identified by NMR. The purity of LIG was examined by HPLC. In this study, LIG ( $>95 \%$ purity) was used and prepared daily in $3 \%(\mathrm{v} / \mathrm{v})$ Tween- 80 (AR, Tianjin Chemical Company, China), and orally administered 20 or $80 \mathrm{mg} / \mathrm{kg}$ at $2 \mathrm{~h}$ after ischemia. Rats in the ischemic control group received the volume-matched vehicle.

Measurement of the Infarct Size Rats were sacrificed under deep re-anesthesia and brains were quickly removed, 
then seven serial coronal sections of 2-mm thickness were prepared. Brain slices were incubated in a $0.2 \%$ solution of 2,3,5-triphenyltetrazolium chloride (TTC, Ameresco U.S.A.) in phosphate buffered saline at $37^{\circ} \mathrm{C}$ for $30 \mathrm{~min}$ and fixed by immersion in $4 \%$ buffered formaldehyde solution overnight. $^{8)}$ The normal area of brain was stained dark red based on intact mitochondrial function whereas infarct tissue remained unstained (white). The area of infarction and of both cerebral hemispheres in each slice were scanned using a color flatbed scanner, and histologically quantified using a computerized image analysis (Pro plus 4.5 version, Image $\mathrm{J}$, Bethesda, MD, U.S.A.). The infarct size was calculated from the sum of the infarct areas (seven sections in all) $\times$ thickness $(2 \mathrm{~mm})$. The degree of infarction was expressed as a percent volumes of the whole coronal section.

Edema The contralateral (non-ischemic) hemispheric volume was used as a control for calculation of ischemia-induced brain edema in the ipsilateral (ischemic) hemisphere. A net difference in hemispheric volumes was gained by subtracting the volume of the hemisphere from that of the infarcted hemisphere. Brain swelling was expressed as a percentage of the net difference in ischemic hemispheric volume over the contralateral hemispheric volume. ${ }^{9)}$

Assessment of Neurological Deficits At $24 \mathrm{~h}$ ischemia, neurological examination was blindly performed according to the neurologic grading system described by Menzies et al. ${ }^{10)}$ : $0=$ no apparent deficits; $1=$ left forelimb flexion; $2=$ decreased grip of the left forelimb while tail pulled; $3=$ spontaneous movement in all directions; left circling only if pulled by tail; $4=$ spontaneous left circling. Meanwhile, the survival rate was recorded at the end of experiment.

Core Temperature Monitoring Rat rectal temperature was monitored with a temperature probe (DT-02C, Jaxun, Hangzhou, China) prior to and after ischemia 2, 3, 4, 6, 7, and $24 \mathrm{~h}$ following the occlusion of MCA. The probe was inserted $3 \mathrm{~cm}$ and took about $30 \mathrm{~s}$ to reach a stable temperature.

Statistical Analysis Results are presented as the mean \pm S.E.M. obtained from 6-7 rats in each group. Data were analyzed by either Student's $t$-test or one-way analysis of variance (ANOVA). $p$ values less than 0.05 were considered statistically significant.

\section{RESULTS}

Effect of LIG on Infarct Size After $24 \mathrm{~h}$ of permanent occlusion of MCA, the ischemic zone was identified in the territory supplied by the MCA as a distinct pale-stained area in the control rats, which was attenuated by treatment with either 20 or $80 \mathrm{mg} / \mathrm{kg} \mathrm{LIG} \mathrm{(Fig.} \mathrm{2).} \mathrm{The} \mathrm{computerized} \mathrm{image}$ analysis indicated that the infarct volumes in control rats treated without LIG were $21.08 \pm 1.82 \%$ of total cerebral volume, which were dose-dependently and significantly reduced to $10.90 \pm 2.11 \%$ and $3.19 \pm 3.00 \%$ in $20 \mathrm{mg} / \mathrm{kg}$ or $80 \mathrm{mg} / \mathrm{kg}$ LIG-treated animals, respectively $(p<0.01 v s$. control, Fig. 3). LIG $(20$, or $80 \mathrm{mg} / \mathrm{kg})$, orally administrated at $2 \mathrm{~h}$ ischemia, reduced the cerebral infarct volumes by $48.29 \%$ and $84.87 \%$ respectively compared to ischemic control group.

Effect of LIG on Brain Edema Twenty-four hours after focal ischemia, the ipsilateral (ischemic) hemisphere showed significantly swelling compared with the contralateral (nonischemic) hemisphere. The average edema percentage in

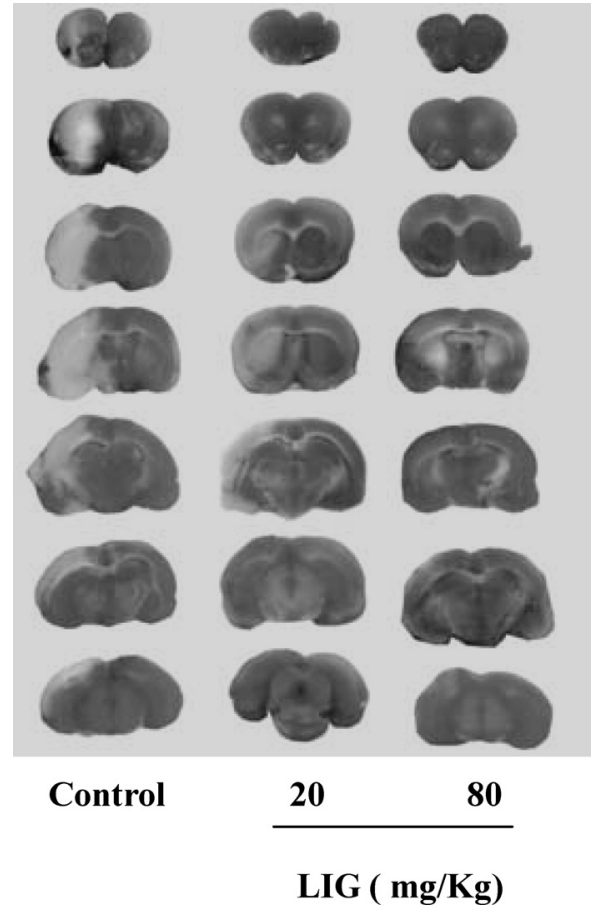

Fig. 2. Illustrative Coronal Sections (2-mm Thick) Showing Infarct Area in the Ischemic Cerebral Hemisphere as Distinct Pale-Stained Area in Rats Subjected to $24 \mathrm{~h}$ MCA Occlusion (Control) and Attenuation of Infarct Area by Oral Administration of LIG $(20$, or $80 \mathrm{mg} / \mathrm{kg})$ at $2 \mathrm{~h}$ Ischemia

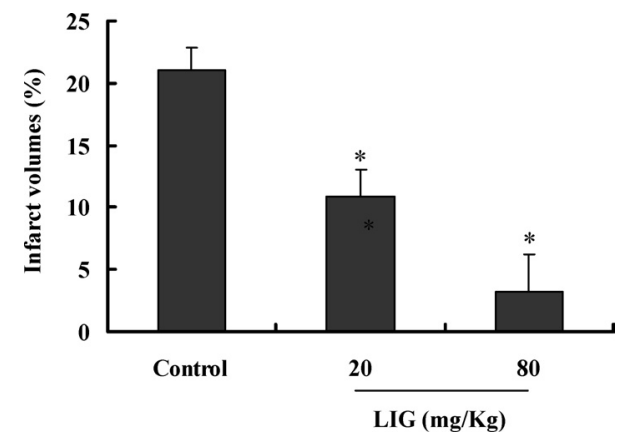

Fig. 3. Effect of LIG on the Infarct Volume of the Whole Coronal Sections in Rats Subjected to $24 \mathrm{~h}$ Occlusion of MCA

Animals received LIG $(20$, or $80 \mathrm{mg} / \mathrm{kg}$, orally) at $2 \mathrm{~h}$ of ischemia. Cerebral Infarction size was expressed as a percent volume of the whole coronal sections. Infarct volumes markedly decreased by treatment with LIG in dose-dependent manner. Data represent the mean \pm S.E.M. of $6-7$ animals. $* p<0.01$ versus control.

ischemic control animals treated without LIG was $15.74 \pm$ $2.91 \%$, which was decreased to $4.94 \pm 1.83 \%$ or $2.82 \pm$ $1.82 \%$, respectively, by the treatment with 20 or $80 \mathrm{mg} / \mathrm{kg}$ LIG ( $p<0.01 v s$. control, Fig. 4). LIG (20, or $80 \mathrm{mg} / \mathrm{kg})$, orally administrated at $2 \mathrm{~h}$ ischemia, reduced the brain edema by $43.4 \%$ and $83.4 \%$ respectively compared to ischemic control group.

Effect of LIG on Neurologial Scores At $24 \mathrm{~h}$ of focal ischemia, the neurological scores in control rats were measured to be $3.63 \pm 0.21$, which were significantly decreased to $2.57 \pm 0.20$ or $1.83 \pm 0.40$, respectively, by the treatment with 20 or $80 \mathrm{mg} / \mathrm{kg}(p<0.01$ vs. control group, Fig. 5). These results demonstrated that oral administration of LIG exhibited a substantial improvement in rat neurobehavioral impairment induced by permanent focal ischemia. LIG treated groups 


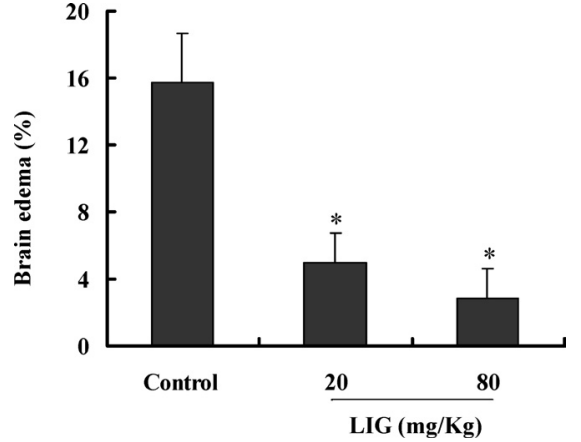

Fig. 4. Effect of LIG on Ischemic Brain Swelling Induced by $24 \mathrm{~h}$ Occlusion of MCA in Rats

Ischemic brain swelling following $24 \mathrm{~h}$ ischemia in vehicle or LIG-treated animals was expressed as a percentage of net difference in the ischemic hemispheric volume over the contralateral hemispheric volume, see text for details. Results are presented as the means \pm S.E.M. from $6-7$ rats in each group. $* p<0.01$ versus control.

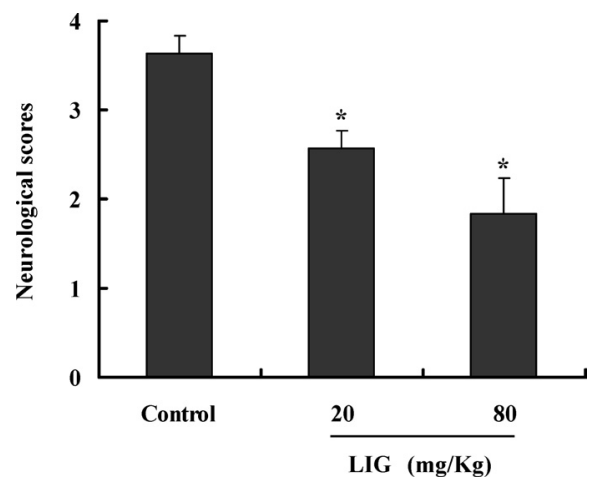

Fig. 5. Effect of LIG on Neurological Deficits Due to $24 \mathrm{~h}$ Occlusion of MCA in Rats

Neurological scores were evaluated as described in the text at $24 \mathrm{~h}$ after ischemia. Data are presented as the means \pm S.E.M. from $6-7$ rats in each group. $* p<0.01$ ver sus control.

had higher survival rates $(77 \%$ or $86 \%$ at the doses of 20 or $80 \mathrm{mg} / \mathrm{kg}$, respectively) at $24 \mathrm{~h}$ after permanent MCA than control group (60\%).

Effect of LIG on Body Temperature The core body temperature, prior to ischemia, had no differences among different groups. Permanent MCA occlusion induced the hyperthermia with about a $2{ }^{\circ} \mathrm{C}$ increase in control rats at the early stage of ischemia compared with the baseline temperature, whereas postischemic treatment with 20 or $80 \mathrm{mg} / \mathrm{kg} \mathrm{LIG}$ had no significant effect on spontaneous hyperthermia in MCA occlusion model (Fig. 6). Treatment with LIG at the used doses had not appeared hypothermic effect.

\section{DISCUSSION}

3-Alkylphthalide derivatives, which exist widely in natural Umbelliferaceae plants, have been shown to possess various bioactive effects. Recently, pharmacological studies of 3- $n$ alkyphthalide derivatives made a great progress. Studies have shown that 3- $n$-butylphthalide (NBP, Fig. 1), a component isolated from the seeds of Apium graveolens LiNN, has many significant protective effects on brain ischemic damage, including reducing the neuronal apoptosis and the infarct volume in transient focal cerebral ischemia in rats, lengthening the life span and improving the neurological deficit in stroke-

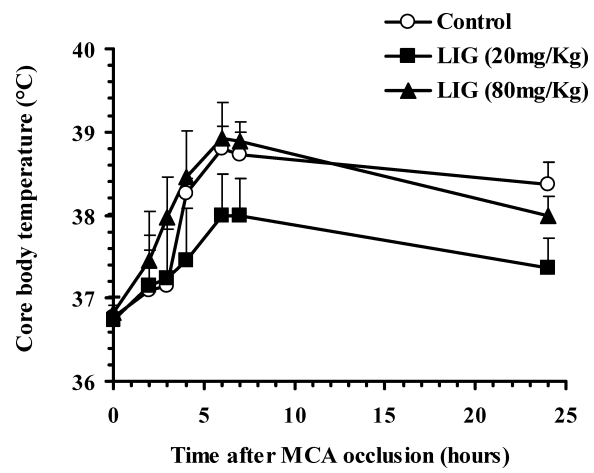

Fig. 6. Effect of LIG on Body Temperature Following the Occlusion of MCA in Rats

Body temperature was recorded in control and LIG-treated groups prior to or after ischemia. Data are presented as the means \pm S.E.M. from 6-7 rats in each group. The temperature curves between control and LIG-treated groups were not statistically different.

prone and spontaneously hypertensive rats. ${ }^{11-13)}$ NBP has been proven effective in clinical trials and approved for the treatment of acute ischemic stroke by the State Food and Drug Administration of China. ${ }^{14,15)}$ LIG is a characteristic phthalide component of Umbelliferae plants such as Danggui and Chuanxiong. We have reported LIG protection against cerebral ischemic damage induced by transient MCA occlusion in rats ${ }^{5)}$ and forebrain ischemia in mice. ${ }^{4)}$ The present study demonstrated, for the first time, the protection by LIG treatment against permanent focal cerebral ischemic damage. LIG $(20$, or $80 \mathrm{mg} / \mathrm{kg})$ treatment, orally administrated at $2 \mathrm{~h}$ ischemia, significantly and dose-dependently reduced cerebral infarct volume and brain edema at $24 \mathrm{~h}$ MCA occlusion. LIG administration also remarkably improved neurological deficit scores and survival rates when tested $24 \mathrm{~h}$ after ischemia.

The mechanisms of LIG protection have not been completely delineated. Our previous study demonstrated that LIG ( 5 , or $20 \mathrm{mg} / \mathrm{kg}$, i.p.), administrated at the start of reperfusion following the bilateral common carotid arteries occlusion, significantly decreased the level of malondialdehyde (MDA) and increased the activities of the antioxidant enzyme glutathione peroxidase (GSH-PX) and superoxide dismutase (SOD) in the transient forebrain ischemic tissues, together with a great up-regulation in Bcl-2 expression and a significant inhibition in Bax and caspase- 3 expression in the ischemic cortex. ${ }^{4)}$ In addition, we have found that LIG (20, or $80 \mathrm{mg} / \mathrm{kg}$ ), orally administrated at the start and $4 \mathrm{~h}$ again following $2 \mathrm{~h}$ MCA occlusion, had significant neuroprotective effect at least partly by the reduction on iNOS expression and NO level in transient focal ischemic brain. ${ }^{5)}$ The above data indicate that the antioxidant effect and the inhibition on both mitochondria-related caspase- 3 apoptotic pathway and iNOS-related pathway contribute to the neuroprotective effect of LIG against cerebral ischemic damage. On the other hand, cerebral ischemia-induced brain edema increases intracranial pressures and then decreases cerebral blood flow, which can aggravate cerebral ischemic outcomes. It is reported that main vasogenic edema is accompanied by an increase in brain water content and extravasation of serum components through blood-brain barrier (BBB) disruption in ischemic stroke. ${ }^{16)}$ And inflammatory mediators, such as TNF- $\alpha$, may lead to the increases in BBB permeability through the NF- $\kappa \mathrm{B}$ dependent signaling process, ${ }^{17)}$ while 
free radicals may directly injury BBB integrity. ${ }^{18)}$ Recent studies indicated that LIG had significantly anti-inflammatory effect probably related to the inhibition of TNF- $\alpha$ and NF- $\kappa$ B activity. ${ }^{19,20)}$ Taken together, the antioxidative and anti-inflammatory activities of LIG may contribute to its improvement on ischemic brain edema, at least in part, by reconstitution of BBB integrity and permeability.

The blood supply to the hypothalamus originates from the anterior cerebral artery, the internal carotid artery, and the posterior communication artery. We detected that there was about $20-30 \%$ decrease of cerebral blood flow measured by a laser-Doppler flowmeter (Periflux 5000, Perimed, Sweden) while a filament was inserted to block the blood flow to rat MCA (Lab data, not shown), which accordingly interrupts the blood flow to the hypothalamus as previously described. ${ }^{21)}$ Postischemic hyperthermia might occur due to the ischemic damage of the hypothalamic temperature regulating center in MCA occlusion model induced by the intraluminal filament technique, ${ }^{21,22)}$ although many factors, including cerebral ischemic duration, types of anesthetics or rat strains, may effect the time points at which body temperature begins to rise. Li showed that a graduate rise in rat body temperature was first observed at $2 \mathrm{~h}$ ischemia and reached highest point at $6 \mathrm{~h}$ in rat MCAO model, which is consistent with our present data. ${ }^{23)}$ It is well known that hyperthermia or fever may increase infarct volumes and worsens the outcome of ischemic stroke through several mechanisms, ${ }^{24,25)}$ whereas hypothermia reducing body temperature to $33^{\circ} \mathrm{C}$ or below might provide dramatic neuroprotection for ischemic stroke. ${ }^{26,27)}$ LIG has been shown to cause hypothermia in an early study. ${ }^{28)}$ It was reported that LIG at 98 or $196 \mathrm{mg} / \mathrm{kg}$ i.p. decreased the body temperature from $37.9 \pm 0.2^{\circ} \mathrm{C}$ and $38.1 \pm 0.2^{\circ} \mathrm{C}$ to $36.8 \pm 0.4^{\circ} \mathrm{C}$ and $35.9 \pm 0.2^{\circ} \mathrm{C}$ in normal mice, respectively. ${ }^{28)}$ In order to determine whether the potential hypothermia of LIG in normal animals was involved in its protection in focal cerebral ischemic lesion, the effect of LIG on the core body temperature was monitored during ischemia. The present results showed that oral administration of 20 , or $80 \mathrm{mg} / \mathrm{kg} \mathrm{LIG} \mathrm{had} \mathrm{no} \mathrm{significant} \mathrm{effect} \mathrm{on} \mathrm{the} \mathrm{in-}$ crease of rat core temperature in permanent focal cerebral ischemia. Hypothermia is therefore unlikely to be associated with LIG protection against cerebral ischemic damage induced by permanent MCA occlusion.

In conclusion, postischemic treatment with LIG significantly decreased the infarct areas and ischemic brain swelling as well as improved neurological behavioral deficits occurring after MCA occlusion. The present data further demonstrate the neuroprotective effect of LIG in permanent focal cerebral ischemia and suggest that LIG may be of therapeutic value in ischemic stroke.

\section{REFERENCES}

1) Warlow C. P., Lancet, 352, SIII1-SIII4 (1998).

2) Du R. J., Bai B., Yu Y., Wang Ch. Y., Qian Ah. M., Zhongguo Zhong Yao Za Zhi, 30, 1400—1406 (2005).

3) Takashi Y., Hokkaidoritsu Eisei Kenkyusyoho, 32, 12-16 (1982).

4) Kuang X., Yao Y., Du J. R., Liu Y. X., Wang Ch. Y., Qian Zh. M., Brain Res., 1102, 145-153 (2006).

5) Zhang G. Y., Du J. R., Kuang X., Yao Y., Liu Y. X., Wang Ch. Y., Qian Zh. M., West China J. Pharm. Sci., 21, 114-117 (2006).

6) Karpiak S. E., Tagliavia A., Wakade C. G., Annu. Rev. Pharmacol. Toxicol., 29, 403-414 (1989).

7) Li Q., Stephenson D., Exp. Neurol., 177, 531—537 (2002).

8) Joshi C. N., Jain S. K., Murthy P. S. R., Brain Res. Protoc., 13, 11-17 (2003).

9) Belayev L., Liu Y., Zhao W., Busto R., Ginsberg M. D., Stroke, 32, $553-560$ (2001).

10) Menzies S. A., Hoff J. T., Betz A. L., Neurosurgery, 31, 100-107 (1992).

11) Chang Q., Wang X. L., Acta Pharmaceut. Sin., 24, 796-804 (2003).

12) Liu X. G., Feng Y. P., Acta Pharmaceut. Sin., 30, 896-903 (1995).

13) Zhang L. Y., Feng Y. P., Acta Pharmaceut. Sin., 31, 18-23 (1996).

14) Cui L. Y., Li S. W., Lu C. Z., Dong Q., Dong P., Shi L. F., Huang J. Zh., Mao Sh. Y., Zhang Ch. D., Cao Y. P., Nie Y. X., Wang W. Zh., Liang Q. Ch., Yang Ch. X., Dong W. W., Li G. Q., Peng G. G., Luo Zh. M., He L., Wang Q., Han J. L., Huang R. X., Li L., Li Ch. Y., Meng Zh. Zh., Chin. J. Cerebrovasc. Dis., 18, 112-115 (2005).

15) Wang H. Y., Huang H. Y., Chin. J. Med. Guide, 5, 448 (2003).

16) Heo H. J., Han S. W., Lee S. K., Free Radic. Biol. Med., 39, 51-70 (2005).

17) Trickler W. J., Mayhan W. G., Miller D. W., Brain Res., 1048, 24-31 (2005).

18) Ding-Zhou L., Marchand-Verrecchia C., Palmier B., Croci N., Chabrier P. E., Plotkine M., Marqaill I., J. Pharmacol. Exp. Ther., 306, 588-594 (2003).

19) Liu L., Ning Z. Q., Shan S., Zhang K., Deng T., Lu X. P., Cheng Y. Y., Planta Med., 71, 808-813 (2005).

20) Yao Y., Du J. R., Bai B., Wang Ch. Y., Qian Zh. M., Nat. Prod. Res. Dev., 15, 751-755 (2006).

21) Zhao Q., Memezawa H., Smith M. L., Siesjo B. K., Brain Res., 649, $253-259$ (1994)

22) Legos J. J., Mangoni A. A., Read S. J., Cambell C. A., Irving E. A., Roberts J., Barone F. C., Parsons A. A., J. Neurosci. Meth., 113, 159166 (2002).

23) Li F., Omae T., Fisher M., Stroke, 30, 2464 -2471 (1999).

24) Chen Q., Chopp M., Bodzin G., Chen H., J. Cereb. Blood. Flow Metab., 13, 389-394 (1993).

25) Dietrich W. D., Halley M., Valdes I., Busto, R., Acta Neuropathol., 81, 615-625 (1991).

26) Corbett D., Hamilton M., Colbourne F., Exp. Neurol., 163, 200-206 (2000).

27) Maier C. M., Ahern K., Cheng M. L., Lee J. E., Yenari M. A., Steinberg G. K., Stroke, 29, 2171-2180 (1998).

28) Xie F. X., Tao J. Y., Shan Xi New Med., 14, 59—62 (1985). 